\title{
Health Outcomes and Cost of Care Among Older Adults with Schizophrenia: A 10-Year Study Using Medical Records across the Continuum of Care
}

\author{
Hugh C. Hendrie, M.B., Ch.B., D.Sc. ${ }^{1,2,3,4}$, Wanzhu Tu, Ph.D. ${ }^{5}$, Rebeka Tabbey, M.S. ${ }^{5}$, \\ Christianna E. Purnell, B.A. ${ }^{3}$, Roberta J. Ambuehl, B.S. ${ }^{3}$, and Christopher M. Callahan, M.D. \\ $1,2,3$ \\ ${ }^{1}$ Indiana University School of Medicine, Indianapolis, IN \\ ${ }^{2}$ Indiana University Center for Aging Research, Indianapolis, IN \\ ${ }^{3}$ Regenstrief Institute, Inc., Indianapolis, IN \\ ${ }^{4}$ Indiana University School of Medicine Department of Psychiatry, Indianapolis, IN \\ ${ }^{5}$ Indiana University School of Medicine Department of Biostatistics, Indianapolis, IN
}

\begin{abstract}
Objectives-The population of older patients with schizophrenia is increasing. This study describes health outcomes, utilization, and costs over 10 years in a sample of older patients with schizophrenia compared to older patients without schizophrenia.

Design, Setting, Participants-An observational cohort study of 31,588 older adults (mean age 70.44 years) receiving care from an urban public health system, including a community mental health center, during 1999-2008. 1635 (5.2\%) were diagnosed with schizophrenia and 757 (2.4\%) had this diagnosis confirmed in the community mental health center. Patients' electronic medical records were merged with Medicare claims, Medicaid claims, the Minimum Dataset, and the Outcome and Assessment Information Set. Information on medication use was not available.

Measurements-Rates of comorbid conditions, health care utilization, costs, and mortality.

Results-Patients with schizophrenia had significantly higher rates of congestive heart failure ( $45.05 \%$ v. $38.84 \%)$, chronic obstructive pulmonary disease $(52.71 \%$ v. $41.41 \%)$, and hypothyroidism $(36.72 \%$ v. $26.73 \%)$ than the patients without schizophrenia $(\mathrm{p}<0.001)$. They had significantly lower rates of cancer $(30.78 \%$ v. $43.18 \%)$ and significantly higher rates of dementia $(64.46 \%$ v. $32.13 \%)$. The patients with schizophrenia had significantly higher mortality risk (HR: 1.25, CI: 1.07-1.47) than the patients without schizophrenia. They also had significantly higher
\end{abstract}

(C) 2012 American Association for Geriatric Psychiatry. Published by Elsevier Inc. All rights reserved.

Corresponding Author: Hugh C. Hendrie, M.B., Ch.B., D.Sc., Indiana University Center for Aging Research, 410 West $10^{\text {th }}$ Street, Suite 2000, Indianapolis, IN 46202, hhendri@iupui.edu, Phone: (317) 423-5591, Fax: (317) 423-5695.

Publisher's Disclaimer: This is a PDF file of an unedited manuscript that has been accepted for publication. As a service to our customers we are providing this early version of the manuscript. The manuscript will undergo copyediting, typesetting, and review of the resulting proof before it is published in its final citable form. Please note that during the production process errors may be discovered which could affect the content, and all legal disclaimers that apply to the journal pertain. 
rates of health care utilization. The mean costs for Medicare and Medicaid were significantly higher for the patients with schizophrenia than for the patients without schizophrenia.

Conclusions-The management of older adult patients with schizophrenia is creating a serious burden for our health care system, requiring the development of integrated models of health care.

\section{Keywords}

comorbidity; mortality; dementia; cancer; nursing home; hospital care

\section{Objective}

In a 1999 consensus statement Jeste and his colleagues predicted an impending crisis in geriatric mental health care during the next decades. (1) This prediction was based in part upon the general population trends, the greater numbers of older adults being diagnosed with late life mental illness and the increasing numbers of patients with serious mental illness now surviving into old age. (1) This latter prediction appeared a little surprising given that most studies of the seriously mentally ill, including patients with schizophrenia and bipolar illness, suggested much higher mortality rates than the non-mentally ill with a reduction in life expectancy from 13 to 30 years. (2) However there is now good evidence that the predictions of Jeste et al are correct. The population of older patients with schizophrenia and other severe mental disabilities is indeed increasing and likely to increase even more in the coming decades. (3-5) The cost of mental health services for these patients has been reported to be particularly high. (6) In an era of strained health care resources, the significance of these trends extends beyond geriatric mental health care and is likely to have public health implications.

These older "survivors" of schizophrenia are now likely to face the consequences of agingrelated illnesses such as cancer, dementia, and cardiovascular disease in addition to their continued disabilities due to their mental illness. Yet there have been few comprehensive studies of the health status of these older patients and the results of these few studies have been inconsistent. $(7-13,6)$. We identified one study of health care utilization among older adults with schizophrenia which included costs. This study, using 1999 claims data from New Hampshire, reported that the greatest per capita expenditures for Medicare and Medicaid were in older patients with schizophrenia and the increase was largely due to nursing home costs. (14) In our previous study of older adults with serious mental illness, which included schizophrenia, the seriously mentally ill had a significantly higher number of emergency room visits and longer hospitalizations over a one year period compared to the non-mentally ill attending the same health care facility. (13) We were unable to identify any published studies that reported longitudinal patterns of health outcomes or costs among a cohort of older adults with schizophrenia.

The purpose of this study is to describe health care outcomes and utilization over 10 years among a sample of older patients with schizophrenia compared to an age matched sample of older patients without schizophrenia. We rely on data assembled for the "Older Adult Transitions Study" funded by the National Institutes of Health. This database was designed 
to describe the patterns of health care across the continuum of care for a large cohort of vulnerable older adults cared for in an urban public health care system.

\section{Methods}

The "Older Adult Transitions Study" was approved by the IUPUI Institutional Review Board and the Center for Medicare and Medicaid Services Privacy Board.

\section{Facilities}

The study site is Wishard Health Services, an urban safety net health care system providing comprehensive services to the uninsured or underinsured in Indianapolis, IN. Wishard Health Services includes a community mental health center that provides mental health services across the lifespan.

\section{Description of Dataset and Study Sample}

The study sample is defined by patients who received care at Wishard Health Services from the years 1999 to 2008 and reached the age of 65 years during that time. Clinical data for these patients were obtained from an electronic medical record that includes, for example, provider-assigned diagnoses, results of diagnostic testing, prescriptions, and utilization. (15) These local data were merged with data from four additional databases throughout the same time period: (1) Medicare claims; (2) Resident Level Minimum Data Set (MDS); (3) Outcome and Assessment Information Set; (OASIS) and (4) Indiana Medicaid claims. Patients' vital status including date of death was available through the Medicare claims data. In addition, cause of death was obtained from the Indiana State Death Certificates which were available until 2006 through the local electronic medical record.

\section{Identifying Older Adults With and Without a Schizophrenia Diagnosis}

The total population of older adults included in the assembled datasets is 33,337 patients. Of these patients, 31,588 reached the age of 65 between the years 1999 and 2008. Patients with schizophrenia were defined in a two-step process to optimize the specificity of the diagnosis. First, all patients diagnosed by any provider with an International Classification of Disease (ICD) code indicating schizophrenia (e.g. ICD-9 code 295.x) in any of the five linked datasets were identified $(n=1635)$. Second, we identified the subset of patients whose diagnosis could be traced directly to a provider in the community mental health center $(\mathrm{n}=757)$. This subset of patients diagnosed with schizophrenia by a mental health specialist constitutes our sample of schizophrenia cases. Patients with schizophrenia diagnosed in the community mental health center were not significantly different in gender or race, but were older (71.1 vs. 70.0$)$.

Patients never assigned an ICD diagnosis consistent with schizophrenia comprised the comparison sample of older adults without schizophrenia $(n=30,831)$.

Comorbid conditions were also identified based on diagnoses and the corresponding ICD codes assigned by providers. The conditions included: arthritis (714., 715.), diabetes (250.), hypertension (HTN) (401.), congestive heart failure (CHF) (428., 398.91), chronic 
obstructive pulmonary disease (COPD) (491., 492., 496.), stroke (433.1, 434.1), coronary artery disease (CAD) (410., 411., 412., 414.), cancer (140.-172. and 174.-239.), liver disease (570., 571., 572., 573.), renal disease (585.), alcohol abuse (303.), substance abuse (304.), thyroid disease (242., 244.) and dementia (290.0-290.43, 291.2, 294.0-294.9, 331.0$331.9,333.0$, and 797). Information on smoking was also obtained from the electronic medical record.

Notably, the date a subject was first diagnosed with any of the above conditions between 1999-2008 is also captured in the database. Thus, we can identify "incident" cases and dates over time for any of the diagnoses described above. This method was used in a recent study of the patterns of health care use among older adults with dementia using this same database. (16) Because we know the date of birth, the date of death, and the censoring date (December 31, 2008), we can also determine the observation window for any given patient in the cohort.

\section{Data Analysis}

The primary purpose of the analysis was to describe the comorbidity, mortality rate, and health care utilization and costs for older adult patients with schizophrenia in comparison with patients without schizophrenia of similar age. We first examined demographic and clinical characteristics of patients with and without schizophrenia. Summary statistics such as means and standard deviations for continuous variables and proportions for categorical variables were presented in a tabular form. Continuous variables were compared using a t test and category variables were compared using the chi square test. Diagnoses of comorbid conditions in patients with and without schizophrenia were compared using logistic regression models adjusting for age, sex, and race. Proportion of cancer diagnoses were additionally adjusted for cigarette smoking. Survival analysis was then conducted to examine effects of schizophrenia on all-cause mortality. Herein, we modeled the time to mortality using proportional hazard model analysis. Patients alive at the end of follow-up were treated as censored in the analysis. The independent variable of primary interest was schizophrenia. To assess the mortality effect of schizophrenia while accommodating the concurrent influences of demographic, lifestyle and other comorbidities, we added the latter characteristics as covariates. Effects of independent variables were then characterized by hazard ratios. Kaplan-Meier estimates of the survival functions were presented graphically. Causes of death as identified in the state death certificates were summarized and tabulated by schizophrenia groups. For care utilization, we focused on the numbers of hospital admissions, length of hospital stay, nursing home placement, and length of nursing home stay. Because the utilization data were expressed as event counts (or number of days), we used log-linear regression models to analyze the observed counts. To account for the different lengths of observation for individual patients, we included offset parameters in the models to indicate the duration of observation for individual patients. Similarly, we reported the mean Medicare and Medicaid costs for the schizophrenia and non-schizophrenia groups. Total Medicare and Medicaid expenditures for the two groups were also reported and compared. All analyses were implemented using SAS software (Version 9.3). P values less than or equal to 0.05 were considered statistically significant. 
To minimize the possible influences of other serious mental health conditions on the study findings, we conducted additional sensitivity analyses by restricting the sample of patients with schizophrenia to those whose diagnoses had been dated before or at the age of 65 years and by excluding from the control group individuals with diagnoses of bipolar disorder (651 subjects; ICD 9 codes 296.0, 296.4, 296.5, 296.6, 296.7, 296.8) or major recurrent depressive disorders (2361 subjects; ICD 9 code 296.3). Results of the sensitivity analysis were then compared with the study findings.

\section{Results}

The mean number of years of follow up for the sample of patients with schizophrenia was $5.66(\mathrm{SD}=3.28)$ and for the sample without schizophrenia it was $6.69(\mathrm{SD}=3.26)$.

Demographics, comorbidities and lifestyle factors were compared between the two samples in Table 1 . The patients with schizophrenia were slightly younger, more likely to be female and more likely to be black. They were significantly more likely to have a history of smoking and a history of substance abuse or excessive alcohol consumption than the patients without schizophrenia. After adjusting for demographics, the sample of patients with schizophrenia had significantly higher rates of CHF, COPD, diabetes, and hypothyroidism. Patients with schizophrenia had significantly lower rates of cancer and renal disease than did patients without schizophrenia. Rates of cancer diagnoses were compared between the groups while adjusting for age, sex, race, and smoking. The cancer rate was significantly lower in patients with schizophrenia (30.8\% vs. $43.2 \%$; logistic regression Wald $\chi^{2}$ [df=1] $=$ $42.5 ; \mathrm{p}<0.001)$. The dementia rate was doubled in patients with schizophrenia as compared to those without schizophrenia $\left(64.5 \%\right.$ vs. $32.1 \% ; \chi_{[d f=1]}^{2}=369.2 ; \mathrm{p}<0.001$. After adjusting for vascular disease and vascular risk factors, smoking, alcohol abuse, hypertension, diabetes, CAD, CHF and stroke the effect of schizophrenia on dementia rates remained highly significant $(\mathrm{p}<0.0001)$, Amongst 488 patients with schizophrenia and dementia, 248 (50.8\%) had Alzheimer diagnoses (ICD-9 of 331.0). Amongst 9906 of the patients without schizophrenia who had dementia, 4673 (47.2\%) had Alzheimer diagnoses. Thus the proportions of Alzheimer disease in the two samples were not statistically different among those with dementia $\left(\chi_{[\mathrm{df}=1]}^{2}=2.5 ; \mathrm{p}=0.1153\right)$.

There was a significant difference in mortality rates between the patients with and without schizophrenia, as shown by the Kaplan-Meier estimates of survival functions in Figure 1. Using proportional hazard models, we further examined the correlates of mortality in our study sample. As shown in Table 2, schizophrenia was a significant risk factor for all-cause mortality. Specifically, the hazard ratio associated with schizophrenia was 1.25 , suggesting a $25 \%$ increase in the risk of death after controlling for the effects of other potential correlates, including diagnoses of cancer, CHF, COPD, liver disease, alcoholism, smoking, and age (HR: $1.25,95 \%$ CI: $1.07-1.47, \mathrm{p}=0.0060$ ). In a stratified analysis of the patients with schizophrenia, the diagnoses of dementia did not significantly increase the risk of mortality (HR: $1.04 ; 95 \%$ CI: $0.68-1.59 ; \chi^{2}{ }_{[\mathrm{df}=1]}=0.03 ; \mathrm{p}=0.860$ ). However, in the patients without schizophrenia, dementia increased the risk of mortality by approximately $30 \%$ (HR: 1.30 , 95\% CI: $\left.1.22-1.39 ; \chi_{[\mathrm{df}=1]}^{2}=66.7 ; \mathrm{p}=<0.001\right)$. 
The primary cause of death for the two samples is shown in Table 3. Patients with schizophrenia were more likely to die of heart disease/vascular disease and pulmonary disease than patients without schizophrenia. They were less likely to die of cancer and other neurological conditions such as epilepsy, Parkinson's disease, hereditary and degenerative nervous system conditions, and other nervous system disorders.

Table 4 shows comparative data on a number of parameters of healthcare utilization, hospital stay, nursing home stay, and home health care. In all of the parameters involving hospital care and nursing home stay the sample of patients with schizophrenia had significantly higher rates of utilization. The percentage of patients receiving home health care did not differ between the samples but within those that did receive it the patients with schizophrenia had a significantly greater number of home health days accrued and greater length of home health care. The mean total cost of care over the time period of the analyses for both Medicare and Medicaid was significantly higher for the patients with schizophrenia.

In additional analyses, when hospital admissions per year are adjusted for all demographics and comorbidities, the difference between patients with and without schizophrenia becomes non-significant ( $\mathrm{p}=0.8106$ ). When total nursing home days are adjusted for demographics and comorbidities the patients with schizophrenia still have significantly higher rates $(\mathrm{p}<$. 0001).

Neither of the two sensitivity analyses, restricting the sample of patients with schizophrenia to those whose diagnoses were dated before or at the age of 65 years or excluding from the sample of patients without schizophrenia those with bipolar illness or recurrent major disorders, led to any significant change in the results of the main analysis.

\section{Conclusions}

To our knowledge, this is the first observational cohort study of older adult patients with schizophrenia spanning an entire decade and including data across the continuum of care. Older adult patients with schizophrenia had a significantly greater risk for mortality than did the older adult patients without schizophrenia even when controlling for comorbid conditions. This finding is consistent with the results from previous studies of younger samples. (17-20) The mean difference in survival time between the older adult patients with and without schizophrenia at the 60th percentile was approximately 34 months. In younger populations, differences in life expectancy have been reported between 13 to 30 years. (21) Thus, even among patients with schizophrenia aged 65 and older, these survivors continue to experience excess mortality. Differences in comorbidity rates between the patients with and without schizophrenia partially explain the differences in mortality, which is consistent with the literature in younger patients. $(2,22,23)$ Some of these differences may be attributed to the greater use of antipsychotic medication, particularly atypical antipsychotics, in the sample of patients with schizophrenia. $(24,25)$ Unfortunately medication use was not available for this analysis. However, even after controlling for the effects of demographic factors and co-morbidities, patients with schizophrenia still had a $25 \%$ increase in mortality risk as opposed to patients without schizophrenia. The differences in causes of death mirrored the differences in pre-mortem diagnoses from the clinical records. Older adult 
patients with schizophrenia were significantly more likely than patients without schizophrenia to die of heart disease and pulmonary disease but significantly less likely to die of cancer.

The differences in rates of cancer between the two populations were striking. Previous reports on cancer rates in patients with schizophrenia have been inconsistent. One 11 year follow up study compared a large sample of patients with schizophrenia with figures from the general population and found higher rates of deaths due to cancer in the population with schizophrenia particularly for breast cancer and lung cancer. Part of the explanation for their findings of higher rates was smoking. Their study population was younger than ours however, with an age of 18-64 years at baseline. (8) Other studies have reported lower rates of cancer in patients with schizophrenia, particularly in cancers of the bowel, and attributed this to the presence of protective genes in the patients with schizophrenia or the effects of antipsychotic medication. (10) One problem with determining cancer rates in patients with schizophrenia is the possibility of low case identification by examining physicians, but the finding of lower rates of cancer as a cause of death in death certificates may provide some additional validation. In our analyses cancer diagnoses were sub-grouped according to anatomical location. No single cancer type difference reached significance between the groups and the lack of difference in lung cancer is particularly notable given the higher rates of smoking in the patients with schizophrenia.

There have been prior reports identifying specific cognitive problems in younger patients with schizophrenia. $(26,27)$ There are fewer studies of the effect of the aging process on cognitive performance in aging patients with schizophrenia. $(28,29)$ One study reported that the trajectory of cognitive decline was no different in community dwelling late middle-aged patients with schizophrenia (mean age 53.1 years) than in healthy controls. (30) However, another recent study concluded that while schizophrenia is associated with cognitive impairment across all age groups, older patients with schizophrenia over the age of 70 experience relatively greater age-associated differences in cognitive functioning than agematched healthy individuals. (11) There have been remarkably few longitudinal studies of patients with schizophrenia which included dementia as an outcome. (9) One study reported that patients with schizophrenia are 2-3 times more likely subsequently to be diagnosed with dementia compared to the general population. (31) In our analyses the rates of dementia in older adult patients with schizophrenia were twice as high as in the patients without schizophrenia (Table 1). The effect of schizophrenia on dementia rates remained highly significant after adjusting for vascular disease and vascular risk factors, smoking, alcohol abuse, hypertension, diabetes, CAD, CHF, and stroke. Previous post mortem studies on older chronically hospitalized patients with schizophrenia and severe cognitive impairment found little evidence of Alzheimer Disease pathology in their brains. (32) In our analysis an equal proportion of patients with dementia also carried the diagnosis of Alzheimer Disease in both the group of patients with schizophrenia and without (patients with dementia and schizophrenia, 50.8\%; patients with dementia but without schizophrenia, 47.2\%). The lack of a significant effect on mortality of the diagnosis of dementia in the patients with schizophrenia (HR: 1.04, 95\% CI: 0.68-1.59) in contrast to the increased effect of a dementia diagnosis in the total sample (HR: $1.29,95 \%$ CI: $1.21-1.38$ ) raises the possibility that at least in some cases physicians are misidentifying patients in these older, difficult-to- 
evaluate, patients with schizophrenia. The percentage of patients with dementia listed as a cause of death did not differ between the samples although dementia is significantly underreported in death certificates. $(33,34)$

In healthcare parameters involving hospital admissions, hospital lengths of stay, nursing home facility use, and nursing home length of stay patients with schizophrenia had significantly greater indicators of utilization than did patients without schizophrenia. These results are similar to previous reports. $(13,35-39)$ When we adjusted for patient characteristics (demographics, comorbidity differences including dementia and life style differences including smoking and alcohol consumption) the differences in hospital use between the two populations were no longer significant. However, even after adjusting for these characteristics there remained a significant difference between patients with and without schizophrenia for nursing home days. There were no significant differences between the samples in the percentage of patients receiving home health care but in those that did, patients with schizophrenia had a greater number of health care days accrued and longer lengths of stay than did the patients without schizophrenia.

The total costs for Medicare and particularly Medicaid were significantly higher for the patients with schizophrenia compared to the patients without (see Table 4). These results are similar to those previously reported for nursing home care by Bartels et al. (14) However, these data highlight the magnitude of Medicaid expenditures for this population perhaps due to a combination of the burden of schizophrenia and dementia with multiple chronic conditions and limited socioeconomic resources. There are no evidence-based models of care for providing integrated psychiatric and medical care for these older adults in ambulatory, inpatient, or long-term care settings. $(40,41)$ Research is needed to better design systems of care to deliver high-quality efficient care to this population across the continuum of care. (40)

There are some significant strengths in this analysis. We believe it represents the most comprehensive evaluation to date of these parameters in older patients with schizophrenia. The innovation of this project is the rare, if not entirely unique, database which provides access to a clinical electronic record coupled with Medicaid and Medicare claims as well as data on nursing home and home healthcare stays. Obtaining information from death certificates was also a valuable addition. In addition we did conduct two sensitivity analyses restricting the sample of patients with schizophrenia to those whose diagnoses were dated before or at the age of 65 years and excluded from the sample of patients without schizophrenia those patients with bipolar illness or recurrent major disorders, neither of which led to any significant change in the results of the main analysis.

The major limitation lies in the use of clinical records rather than direct examination, particularly with diagnoses such as schizophrenia and dementia. The results must also be interpreted in light of the methods we used to assemble this observational cohort. First, patients had to survive to age 65 . Second, patients do not receive standardized evaluations at standardized intervals over time as one might find with a prospective longitudinal cohort study. This project relies on the naturalistic observational data of a cohort of patients cared 
for in a single health care system. Thus, the data are an accurate depiction of the health care that these patients received in a real-world clinical setting.

This analysis reinforces the concept that the increasing numbers of older seriously mentally ill, particularly older patients with schizophrenia will create a serious burden for our health system which will require the development of new integrated models of health care involving links between the health and mental health systems. The possible link between increased incidence of dementia in older patients with schizophrenia is particularly disturbing and justifies further comprehensive study.

\section{Acknowledgments}

Conflicts of Interest and Source of Funding: This work was supported by the National Institutes of Health (R24 MH080827 and R01 AG031222). All authors were supported by an institution which received funding from these two grants.

\section{References}

1. Jeste DV, Alexopoulos GS, Bartels SJ, et al. Consensus statement on the upcoming crisis in geriatric mental health: research agenda for the next 2 decades. Arch Gen Psychiatry. 1999; 56:848-853. [PubMed: 12884891]

2. De Hert M, Cohen D, Bobes J, et al. Physical illness in patients with severe mental disorders. II. Barriers to care, monitoring and treatment guidelines, plus recommendations at the system and individual level. World Psychiatry. 2011; 10:138-151. [PubMed: 21633691]

3. Harvey PD, Czaja SJ, Loewenstein DA. Schizophrenia in later life. Am J Geriatr Psychiatry. 2012; 20:1-4. [PubMed: 22104378]

4. Cohen CI, Cohen GD, Blank K, et al. Schizophrenia and older adults. An overview: directions for research and policy. Am J Geriatr Psychiatry. 2000; 8:19-28. [PubMed: 10648291]

5. Howard RJ. Schizophrenia in later life: emerging from the shadows. Am J Geriatr Psychiatry. 2010; 18:859-861. [PubMed: 20808149]

6. Cuffel BJ, Jeste DV, Halpain M, et al. Treatment costs and use of community mental health services for schizophrenia by age cohorts. Am J Psychiatry. 1996; 153:870-876. [PubMed: 8659608]

7. Osborn DP, Levy G, Nazareth I, et al. Relative risk of cardiovascular and cancer mortality in people with severe mental illness from the United Kingdom's General Practice Rsearch Database. Arch Gen Psychiatry. 2007; 64:242-249. [PubMed: 17283292]

8. Tran E, Rouillon F, Loze JY, et al. Cancer mortality in patients with schizophrenia: an 11-year prospective cohort study. Cancer. 2009; 115:3555-3562. [PubMed: 19548261]

9. Shah JN, Qureshi SU, Jawaid A, et al. Is There Evidence for Late Cognitive Decline in Chronic Schizophrenia? Psychiatr Q. 2011

10. Tabares-Seisdedos R, Dumont N, Baudot A, et al. No paradox, no progress: inverse cancer comorbidity in people with other complex diseases. Lancet Oncol. 2011; 12:604-608. [PubMed: 21498115]

11. Loewenstein DA, Czaja SJ, Bowie CR, et al. Age-associated differences in cognitive performance in older patients with schizophrenia: a comparison with healthy older adults. Am J Geriatr Psychiatry. 2012; 20:29-40. [PubMed: 22130385]

12. Chou FH, Tsai KY, Su CY, et al. The incidence and relative risk factors for developing cancer among patients with schizophrenia: a nine-year follow-up study. Schizophr Res. 2011; 129:97103. [PubMed: 21458957]

13. Hendrie HC, Hay D, Lane K, et al. Comorbidity profile and health care utilization in elderly patients with serious mental illnesses. American Journal of Geriatric Psychiatry. in press;

14. Bartels SJ, Clark RE, Peacock WJ, et al. Medicare and medicaid costs for schizophrenia patients by age cohort compared with costs for depression, dementia, and medically ill patients. Am J Geriatr Psychiatry. 2003; 11:648-657. [PubMed: 14609805] 
15. McDonald CJ, Overhage JM, Tierney WM, et al. The Regenstrief Medical Record System: a quarter century experience. Int J Med Inform. 1999; 54:225-253. [PubMed: 10405881]

16. Callahan CM, Arling G, Tu W, et al. Transitions in Care among Older Adults with and without Dementia. Journal of the American Geriatrics Society. in press;

17. Brown S, Kim M, Mitchell C, et al. Twenty-five year mortality of a community cohort with schizophrenia. Br J Psychiatry. 2010; 196:116-121. [PubMed: 20118455]

18. Chwastiak LA, Rosenheck RA, Desai R, et al. Association of psychiatric illness and all-cause mortality in the National Department of Veterans Affairs Health Care System. Psychosom Med. 2010; 72:817-822. [PubMed: 20639387]

19. Laursen TM, Munk-Olsen T, Nordentoft M, et al. Increased mortality among patients admitted with major psychiatric disorders: a register-based study comparing mortality in unipolar depressive disorder, bipolar affective disorder, schizoaffective disorder, and schizophrenia. J Clin Psychiatry. 2007; 68:899-907. [PubMed: 17592915]

20. Saha S, Chant D, McGrath J. A systematic review of mortality in schizophrenia: is the differential mortality gap worsening over time? Arch Gen Psychiatry. 2007; 64:1123-1131. [PubMed: 17909124]

21. Colton CW, Manderscheid RW. Congruencies in increased mortality rates, years of potential life lost, and causes of death among public mental health clients in eight states. Prev Chronic Dis. 2006; 3:A42. [PubMed: 16539783]

22. Weber NS, Cowan DN, Millikan AM, et al. Psychiatric and general medical conditions comorbid with schizophrenia in the National Hospital Discharge Survey. Psychiatr Serv. 2009; 60:10591067. [PubMed: 19648193]

23. Carney CP, Jones L, Woolson RF. Medical comorbidity in women and men with schizophrenia: a population-based controlled study. J Gen Intern Med. 2006; 21:1133-1137. [PubMed: 17026726]

24. Kraemer S, Minarzyk A, Forst T, et al. Prevalence of metabolic syndrome in patients with schizophrenia, and metabolic changes after 3 months of treatment with antipsychotics--results from a German observational study. BMC Psychiatry. 2011; 11:173. [PubMed: 22044502]

25. Mitchell AJ, Vancampfort D, Sweers K, et al. Prevalence of Metabolic Syndrome and Metabolic Abnormalities in Schizophrenia and Related Disorders--A Systematic Review and Meta-Analysis. Schizophr Bull. 2011

26. Keefe RS, Bilder RM, Harvey PD, et al. Baseline neurocognitive deficits in the CATIE schizophrenia trial. Neuropsychopharmacology. 2006; 31:2033-2046. [PubMed: 16641947]

27. Heinrichs RW. The primacy of cognition in schizophrenia. Am Psychol. 2005; 60:229-242. [PubMed: 15796677]

28. Andreasen NC, Nopoulos P, Magnotta V, et al. Progressive brain change in schizophrenia: a prospective longitudinal study of first-episode schizophrenia. Biol Psychiatry. 2011; 70:672-679. [PubMed: 21784414]

29. Jarskog LF, Miyamoto S, Lieberman JA. Schizophrenia: new pathological insights and therapies. Annu Rev Med. 2007; 58:49-61. [PubMed: 16903799]

30. Nayak Savla G, Moore DJ, Roesch SC, et al. An evaluation of longitudinal neurocognitive performance among middle-aged and older schizophrenia patients: use of mixed-model analyses. Schizophr Res. 2006; 83:215-223. [PubMed: 16507344]

31. Korner A, Lopez AG, Lauritzen L, et al. Late and very-late first-contact schizophrenia and the risk of dementia--a nationwide register based study. Int J Geriatr Psychiatry. 2009; 24:61-67. [PubMed: 18561206]

32. Arnold SE, Trojanowski JQ. Cognitive impairment in elderly schizophrenia: a dementia (still) lacking distinctive histopathology. Schizophr Bull. 1996; 22:5-9. [PubMed: 8685663]

33. Kuller LH, Ives DG. Vital records and dementia. Neuroepidemiology. 2009; 32:70-71. [PubMed: 19001799]

34. Zilkens RR, Spilsbury K, Bruce DG, et al. Linkage of hospital and death records increased identification of dementia cases and death rate estimates. Neuroepidemiology. 2009; 32:61-69. [PubMed: 19001798] 
35. Andrews AO, Bartels SJ, Xie H, et al. Increased risk of nursing home admission among middle aged and older adults with schizophrenia. Am J Geriatr Psychiatry. 2009; 17:697-705. [PubMed: 19625787]

36. Miller EA, Rosenheck RA. Risk of nursing home admission in association with mental illness nationally in the Department of Veterans Affairs. Med Care. 2006; 44:343-351. [PubMed: 16565635]

37. Fullerton CA, McGuire TG, Feng Z, et al. Trends in mental health admissions to nursing homes 1999-2005. Psychiatr Serv. 2009; 60:965-971. [PubMed: 19564228]

38. Aschbrenner K, Grabowski DC, Cai S, et al. Nursing home admissions and long-stay conversions among persons with and without serious mental illness. J Aging Soc Policy. 2011; 23:286-304. [PubMed: 21740203]

39. Mai Q, Holman CD, Sanfilippo FM, et al. The impact of mental illness on potentially preventable hospitalisations: a population-based cohort study. BMC Psychiatry. 2011; 11:163. [PubMed: 21985082]

40. Bartels SJ, Forester B, Mueser KT, et al. Enhanced skills training and health care management for older persons with severe mental illness. Community Ment Health J. 2004; 40:75-90. [PubMed: 15077730]

41. Tosh G, Clifton A, Mala S, et al. Physical health care monitoring for people with serious mental illness. Cochrane Database Syst Rev. 2010 CD008298. 


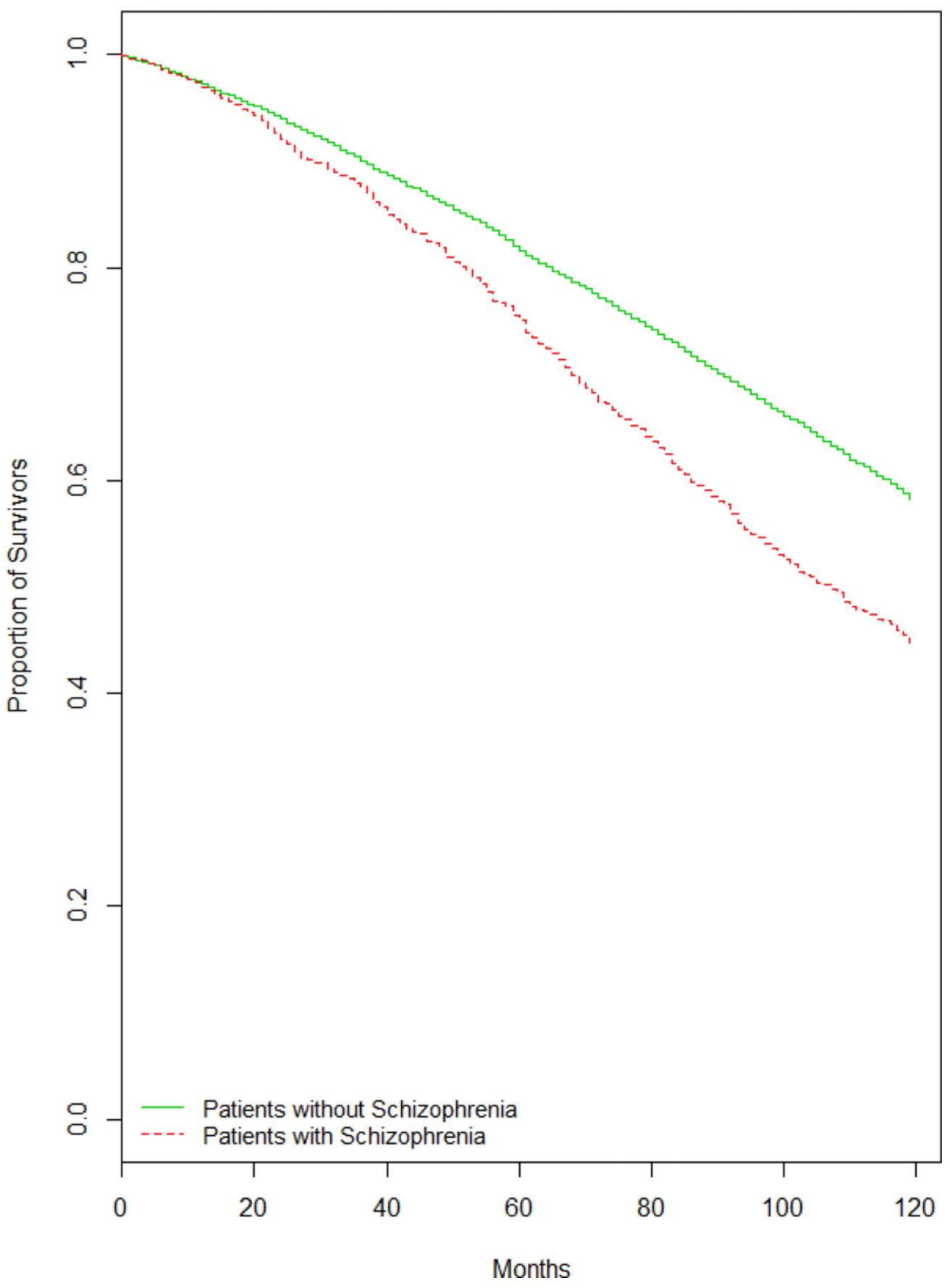

Figure 1.

Kaplan-Meier Estimates of the Proportion of Mortality as a Function of Time for Patients With and Without Schizophrenia 
Table 1

Demographics and Clinical Characteristics of Patients With and Without Schizophrenia Adjusted for Age, Gender, and Ethnicity.

\begin{tabular}{|c|c|c|c|c|}
\hline & $\begin{array}{r}\text { Total Sample } \\
\mathbf{n}=\mathbf{3 1}, \mathbf{5 8 8}\end{array}$ & $\begin{array}{r}\text { Patients with } \\
\text { Schizophrenia } \\
\mathbf{N}=757\end{array}$ & $\begin{array}{r}\text { Patients } \\
\text { without } \\
\text { Schizophrenia } \\
\mathbf{N}=\mathbf{3 0 , 8 3 1}\end{array}$ & P-value \\
\hline \multicolumn{5}{|l|}{ Demographics } \\
\hline Age, mean (SD) & $70.44(6.81)$ & $69.96(6.46)$ & $70.46(6.82)$ & 0.048 \\
\hline Female, $\%$ & 59.25 & 69.22 & 59.00 & $<.001$ \\
\hline Black, \% & 35.47 & 40.82 & 35.34 & 0.002 \\
\hline \multicolumn{5}{|l|}{ Comorbid conditions } \\
\hline Arthritis, \% & 57.97 & 58.26 & 57.96 & 0.317 \\
\hline Coronary Artery Disease, $\%$ & 47.96 & 49.67 & 47.92 & 0.179 \\
\hline Cancer, $\%$ & 42.88 & 30.78 & 43.18 & $<.001$ \\
\hline Congestive Heart Failure, $\%$ & 38.99 & 45.05 & 38.84 & $<.001$ \\
\hline Chronic Obstructive & 41.68 & 52.71 & 41.41 & $<.001$ \\
\hline \multicolumn{5}{|l|}{ Pulmonary Disease, $\%$} \\
\hline Dementia, $\%$ & 32.90 & 64.46 & 32.13 & $<.001$ \\
\hline Diabetes, $\%$ & 45.83 & 55.75 & 45.59 & $<.001$ \\
\hline Alcohol Abuse, $\%$ & 5.01 & 14.40 & 4.78 & $<.001$ \\
\hline Ever Smoked, \% & 48.50 & 56.56 & 48.02 & 0.003 \\
\hline Hypertension, \% & 84.72 & 86.92 & 84.67 & 0.462 \\
\hline Hyperthyroid, \% & 7.47 & 8.59 & 7.45 & 0.689 \\
\hline Hypothyroid, \% & 26.97 & 36.72 & 26.73 & $<.001$ \\
\hline Liver Disease, $\%$ & 14.31 & 12.81 & 14.35 & 0.164 \\
\hline Renal Disease, \% & 5.43 & 3.96 & 5.46 & 0.021 \\
\hline Stroke, $\%$ & 17.69 & 17.97 & 17.68 & 0.991 \\
\hline Substance Abuse, $\%$ & 1.38 & 5.42 & 1.28 & $<.001$ \\
\hline
\end{tabular}

Notes: Comparisons of age, and gender and race were based on two sample t test and chi-square tests, respectively; comparisons of comorbidities (except for cancer) were conducted using logistic regression, adjusting for age, gender, and race. The p values were based on the Wald test with one degree of freedom. Cancer prevalence rates in the two groups were compared using logistic regression model adjusting for age, gender, race, and smoking status. We performed a logistic regression analysis on dementia diagnoses, additionally adjusting for the effects of vascular diseaserelated risk factors, schizophrenia remained a significant factor in the analysis. 


\section{Table 2}

Demographic and Clinical Correlates of Mortality

\begin{tabular}{|lrrr|}
\hline Variable & $\begin{array}{r}\text { Hazard } \\
\text { Ratio }\end{array}$ & $\mathbf{9 5 \%}$ CI & $\begin{array}{r}\text { P- } \\
\text { Value }\end{array}$ \\
Dementia & 1.29 & $(1.21,1.38)$ & $<.0001$ \\
Cancer & 1.32 & $(1.25,1.39)$ & $<.0001$ \\
Coronary Artery Disease & 1.05 & $(0.98,1.12)$ & 0.1373 \\
Congestive Heart Failure & 1.72 & $(1.61,1.83)$ & $<.0001$ \\
Hypertension & 0.67 & $(0.60,0.74)$ & $<.0001$ \\
Arthritis & 0.58 & $(0.54,0.61)$ & $<.0001$ \\
Diabetes & 1.04 & $(0.98,1.10)$ & 0.2141 \\
Hyperthyroid & 0.96 & $(0.86,1.06)$ & 0.4066 \\
Chronic Obstructive Pulmonary Disease & 1.28 & $(1.20,1.36)$ & $<.0001$ \\
Liver Disease & 1.17 & $(1.09,1.26)$ & $<.0001$ \\
Stroke & 1.02 & $(0.95,1.09)$ & 0.5886 \\
Hypothyroid & 0.81 & $(0.76,0.87)$ & $<.0001$ \\
Renal Disease & 0.96 & $(0.88,1.05)$ & 0.3961 \\
Alcohol Abuse & 1.20 & $(1.09,1.33)$ & 0.0003 \\
Substance Abuse & 1.20 & $(0.97,1.49)$ & 0.0909 \\
Ever Smoked & 1.60 & $(1.51,1.70)$ & $<.0001$ \\
Black & 0.97 & $(0.91,1.02)$ & 0.2258 \\
Female & 0.84 & $(0.79,0.89)$ & $<.0001$ \\
Baseline Age & 1.06 & $(1.06,1.07)$ & $<.0001$ \\
Schizophrenia & 1.25 & $(1.07,1.47)$ & 0.0060 \\
\hline
\end{tabular}

Notes: The proportional hazard model was fitted using SAS PROC PHREG. P values were based on the Wald test with one degree of freedom. 
Table 3

Causes of Death as Identified in the State Death Certificates to 2006

\begin{tabular}{|lrrrr|}
\hline Cause of Death & $\begin{array}{r}\text { Total Sample } \\
\text { N=6,332 }\end{array}$ & $\begin{array}{r}\text { Patients with } \\
\text { Schizophrenia } \\
\text { N=296 }\end{array}$ & $\begin{array}{r}\text { Patients without } \\
\text { Schizophrenia } \\
\text { N=9,806 }\end{array}$ & $\begin{array}{r}\text { Fisher's Exact } \\
\text { P-Value }\end{array}$ \\
Alcohol Drug Abuse, n (\%) & & $3(1.48)$ & $50(0.82)$ & 0.2410 \\
Anemia, n (\%) & $53(0.84)$ & $0(0.00)$ & $47(0.77)$ & 0.4050 \\
Cancer, n (\%) & $47(0.74)$ & $30(14.78)$ & $1387(22.63)$ & 0.0080 \\
Dementia, n (\%) & $1417(22.38)$ & $15(7.39)$ & $423(6.90)$ & 0.7780 \\
Diabetes, n (\%) & $438(6.92)$ & $10(4.93)$ & $315(5.14)$ & 1.0000 \\
External Injury, n (\%) & $325(5.13)$ & $1(0.49)$ & $134(2.19)$ & 0.1330 \\
HIV, n (\%) & $135(2.13)$ & $0(0.00)$ & $3(0.05)$ & 1.0000 \\
Heart Disease/Vascular Disease, n (\%) & $1773(28.00)$ & $70(34.48)$ & $1703(27.79)$ & 0.0390 \\
Infection, n (\%) & $443(7.00)$ & $11(5.42)$ & $432(7.05)$ & 0.4830 \\
Liver, n (\%) & $49(0.77)$ & $1(0.49)$ & $48(0.78)$ & 1.0000 \\
Neurological, n (\%) & $223(3.52)$ & $1(0.49)$ & $222(3.62)$ & 0.0100 \\
Other, n (\%) & $274(4.33)$ & $13(6.40)$ & $261(4.26)$ & 0.1570 \\
Pulmonary, n (\%) & $626(9.89)$ & $33(16.26)$ & $593(9.68)$ & 0.0040 \\
Renal, n (\%) & $196(3.10)$ & $6(2.96)$ & $190(3.10)$ & 1.0000 \\
Stroke, n (\%) & $330(5.21)$ & $9(4.43)$ & $321(5.24)$ & 0.7480 \\
\hline
\end{tabular}


Table 4

Healthcare Utilization and Healthcare Costs of Patients With and Without Schizophrenia

\begin{tabular}{|c|c|c|c|c|}
\hline & $\begin{array}{r}\text { Total Sample } \\
(\mathbf{n}=\mathbf{3 1 , 5 8 8})\end{array}$ & $\begin{array}{r}\text { Patients with } \\
\text { Schizophrenia } \\
(\mathrm{n}=757)\end{array}$ & $\begin{array}{r}\text { Patients without } \\
\text { Schizophrenia } \\
(\mathrm{n}=\mathbf{3 0 , 8 3 1})\end{array}$ & P-value \\
\hline \multicolumn{5}{|l|}{ Utilization } \\
\hline Subjects with any hospital stay, $\%$ & 63.64 & 77.28 & 63.30 & $<.001$ \\
\hline Total hospital days accrued, mean, (SD) & $31.91(46.54)$ & $58.98(94.17)$ & $31.10(44.08)$ & $<.001$ \\
\hline Hospital admissions per year per 1000 patients & 410 & 550 & 400 & $<.001$ \\
\hline Hospital days per 1000 patients per year & 2,360 & 5,290 & 2,290 & $<.001$ \\
\hline Subjects with any nursing facility use, $\%$ & 31.56 & 56.41 & 30.95 & $<.001$ \\
\hline Total nursing home days accrued, mean, (SD) & $116.31(370.93)$ & $479.47(802.39)$ & $107.39(349.09)$ & $<.001$ \\
\hline Nursing facility days per 1000 patients per year & 16,600 & 68,580 & 15,370 & $<.001$ \\
\hline Nursing facility length of stay, mean, (SD) & $159.55(355.54)$ & $471.34(922.22)$ & $145.59(299.24)$ & $<.001$ \\
\hline Subjects with any home health care use, $\%$ & 33.37 & 31.31 & 33.42 & 0.224 \\
\hline Total home health care days accrued, mean (SD) & $57.11(206.70)$ & $74.12(243.70)$ & $56.69(205.70)$ & 0.092 \\
\hline Home health care days per 1000 patients per year & 8,290 & 10,820 & 8,230 & 0.046 \\
\hline Home health care length of stay, mean (SD) & $79.21(490.20)$ & $78.55(490.38)$ & $107.79(482.33)$ & $<.001$ \\
\hline \multicolumn{5}{|l|}{ Healthcare Cost } \\
\hline Medicare, mean (SD) & $50153.19(70894.88)$ & $63335.01(78866.89)$ & $49829.53(70658.32)$ & $<.001$ \\
\hline Medicaid, mean (SD) & $22655.95(76891.31)$ & $130954.01(198627.40)$ & $19996.80(69245.28)$ & $<.001$ \\
\hline
\end{tabular}

Note: Care utilization measures were analyzed using log-linear models. P values were based on the Wald test with one degree of freedom. Cost was analyzed using t test. 